\title{
Horizontal gene transfer and diverse functional constrains within a common replication-partitioning system in Alphaproteobacteria: the repABC operon
}

\author{
Santiago Castillo-Ramírez*, Jorge F Vázquez-Castellanos, Víctor González \\ and Miguel A Cevallos*
}

Address: Programa de Genómica Evolutiva, Centro de Ciencias Genómicas, Universidad Nacional Autónoma de México, Apartado Postal 565-A, CP 62210, Cuernavaca, Morelos, México

Email: Santiago Castillo-Ramírez* - iago@ccg.unam.mx; Jorge F Vázquez-Castellanos - jvazquez@lcg.unam.mx;

Víctor González - vgonzal@ccg.unam.mx; Miguel A Cevallos* - mac@ccg.unam.mx

* Corresponding authors

Published: 18 November 2009

BMC Genomics 2009, 10:536 doi:10.1 I86/147I-2164-10-536

This article is available from: http://www.biomedcentral.com//47/-2/64//0/536

(C) 2009 Castillo-Ramírez et al; licensee BioMed Central Ltd.

This is an Open Access article distributed under the terms of the Creative Commons Attribution License (http://creativecommons.org/licenses/by/2.0), which permits unrestricted use, distribution, and reproduction in any medium, provided the original work is properly cited.

\begin{abstract}
Background: The repABC plasmid family, which is extensively present within Alphaproteobacteria, and some secondary chromosomes of the Rhizobiales have the particular feature that all the elements involved in replication and partitioning reside within one transcriptional unit, the repABC operon. Given the functional interactions among the elements of the repABC operon, and the fact that they all reside in the same operon, a common evolutionary history would be expected if the entire operon had been horizontally transferred. Here, we tested whether there is a common evolutionary history within the repABC operon. We further examined different incompatibility groups in terms of their differentiation and degree of adaptation to their host.
\end{abstract}

Results: We did not find a single evolutionary history within the repABC operon. Each protein had a particular phylogeny, horizontal gene transfer events of the individual genes within the operon were detected, and different functional constraints were found within and between the Rep proteins. When different repABC operons coexisted in the same genome, they were well differentiated from one another. Finally, we found different levels of adaptation to the host genome within and between repABC operons coexisting in the same species.

Conclusion: Horizontal gene transfer with conservation of the repABC operon structure provides a highly dynamic operon in which each member of this operon has its own evolutionary dynamics. In addition, it seems that different incompatibility groups present in the same species have different degrees of adaptation to their host genomes, in proportion to the amount of time the incompatibility group has coexisted with the host genome.

\section{Background}

The repABC plasmids are a typical genome component of many Alphaproteobacteria species. In fact, more than 20 Alphaproteobacteria species have at least one repABC plas- mid (see refs $[1,2]$ for recent reviews), these repABC plasmids may be the commonest plasmids in Alphaproteobacteria species. In some species these repABC plasmids constitute a significant amount of the bacterial 
genome; such is the case of Rhizobium leguminosarum 3841 , in which repABC plasmids account for 35\% of the genome [3]. This plasmid family includes several incompatibility groups, meaning that more than one type of repABC plasmid can reside in the same bacterial species $[1,2]$. For instance, Rhizobium etli CFN42 has 6 plasmids, all of them repABC plasmids [4]. In contrast to other low copy-number plasmids, in which the elements involved in plasmid replication and segregation are located on different loci (each one under its own regulatory circuit), the repABC plasmids contain all the elements required for replication and partition within the repABC operon. In general, this transcriptional unit comprises three proteinencoding genes (repA, repB, and repC) and a gene encoding a small antisense RNA (ctRNA) [5], which is located within the repB-repC intergenic region. The proteins encoded in the repABC operon have an intricate relationship, with RepA and RepB interacting both with themselves and with each other. These proteins, in conjunction with the centromere-like sequence, parS, function as the plasmid's segregation machinery $[1,2,6]$. On one hand, RepA is a transcriptional repressor of the operon, while RepB acts as its co-repressor by contacting the operator sequence. The third protein-encoding gene of the operon, $r e p C$, is essential for plasmid replication; it encodes the initiator protein, RepC, which exerts its function by binding the origin of replication located within its own coding sequence $[1,2,6]$. Taking these observations into account, it is reasonable to hypothesize that the repABC operon is under concerted evolutionary pressures aimed at maintaining its functionality and avoiding incompatibility with other repABC operons. Remarkably, this operon is not only the replication system of repABC plasmids, but of some secondary chromosomes of some Rhizobiales species. For instance, the second chromosomes of Agrobacterium vitis S4 and Agrobacterium tumefaciens C58 have a repABC origin of replication [7].

At the structural level, the various $r e p A B C$ operons are only superficially homogeneous; they are highly diverse in DNA sequence, and some possess specific structural elements shared only by few members of the family. These distinctive elements fall into three types: (a) the number and class of regulatory elements involved in operon transcription; (b) the number and position of centromere-like sequences (parS sequences); and (c) the presence of peptide-encoding minigenes [1]. Several Alphaproteobacteria genomes possess repAB genes that are not in close association with the ctRNA or repC sequences. However, it has been shown that replication of some Alphaproteobacteria plasmids depends only on RepC and a ctRNA, without the involvement of the repAB genes. This suggests that fusion of different modules could participate in the generation of new repABC plasmids, indicating that the different ele- ments may have experienced different evolutionary histories.

Plasmid stability requires an exquisite balance among all of the interacting molecules involved in plasmid replication and segregation. Perturbation of this balance, for example by the introduction of any replication or segregation element in excess, could lead to plasmid incompatibility. It has been shown that repABC plasmids contain at least four elements involved in plasmid incompatibility: the RepA and RepB proteins, the small antisense RNA, and the parS sequences [6,8-10]. Phylogenies made with RepA, RepB, and RepC proteins have shown that different replicons residing in the same bacterial strain tend to belong to different clades [11]. Other study found that phylogenetic analyses of repABC gene lineages had a lack of evolutionary congruence with the species tree [7]. These observations suggest that divergent evolution followed by episodes of horizontal transfer have played a central role in originating new incompatibility groups. We might therefore expect that incompatibility groups residing in the same genome would be different enough so as to not interfere with each other.

In this study, we analyzed three aspects of repABC operons. First, because it is known that repABC operon has been horizontally transferred, through phylogenetic analyses, we examined horizontal gene transfer of entire operon versus horizontal transfer of individual genes within this operon. This is a key point, since a previous study has shown that some bacterial operons present horizontal gene transfer events that affect not the entire operons but single genes within the operons [12]. Second, we determined the degree of differentiation among repABC operons from different plasmids residing in the same strain (which implies different incompatibility groups). Third, we established the degree of evolutionary adaptedness among different $r e p A B C$ operons coexisting in a single species. In principle, because all the elements of the partition and replication systems are contained in the same operon and the encoded proteins interact, these elements might be expected to present almost the same history. Contrary to this, we found significantly different histories for the various elements of the repABC operon. Moreover, we detected different selective constraints among the elements composing the operon, and even within individual components. As expected, when different incompatibility groups coexisted in a species, these groups were clearly differentiated from one another. Finally, we found different levels of adaptation to the host genome within and between repABC operons coexisting in the same species. 


\section{Results}

\section{The collection of homologous repABC operons}

To date, at least 81 repABC operons have been recognized across the class Alphaproteobacteria [1]. Because we wanted to utilize only homologous groups with the same domain structure, we established strict criteria for defining homologous rep genes and operons (see Methods). As a result of this, we analyzed only 49 operons herein (see Additional file 1). Twenty-one genomes had at least one repABC operon, and most of the operons were located on plasmids. A few genomes, such as those from genera Brucella and Agrobacterium, had repABC operons located on replicons that are considered secondary chromosomes (see Additional file 1). Two Rhizobium species, R. etli CFN42 and $R$. leguminosarum 3841, had the highest number of repABC operons, with seven operons each. All plasmids from these species had a single operon, with the exceptions of plasmid p42f from $R$. etli CFN42 and plasmid pRL11 from R. leguminosarum 3841, which each had two operons per plasmid. We also found six faulty operons that were missing one of the three protein-encoding genes; five out of six were composed of repA and repB genes, while the remaining one consisted of repA and repC. In four of six cases, the faulty operons coexisted with complete operons. In many species only one gene was present; by far the most widely distributed gene was repA, followed by repC (see Additional file 1).

\section{There is no a single history for the repABC operon}

Our first goal was to test whether the elements of the rep $A B C$ operon have a common evolutionary history. A single history would be expected if the entire operon had been transferred; on the opposite, if the individual genes were transferred, several histories would be expected. Given that the partition and replication elements functionally interact with each other and compose a single transcriptional unit, we expected to find a single history $a$ priori. To test this possibility, we constructed individual Bayesian phylogenies for each protein, and used the phylogenies to construct a strict consensus tree. We obtained phylogenies with strong support, but no two phylogenies gave the same topology (see Figure 1). For example, when we considered the phylogenies for RepA and RepC, only five nodes out of 40 achieved a posterior probability

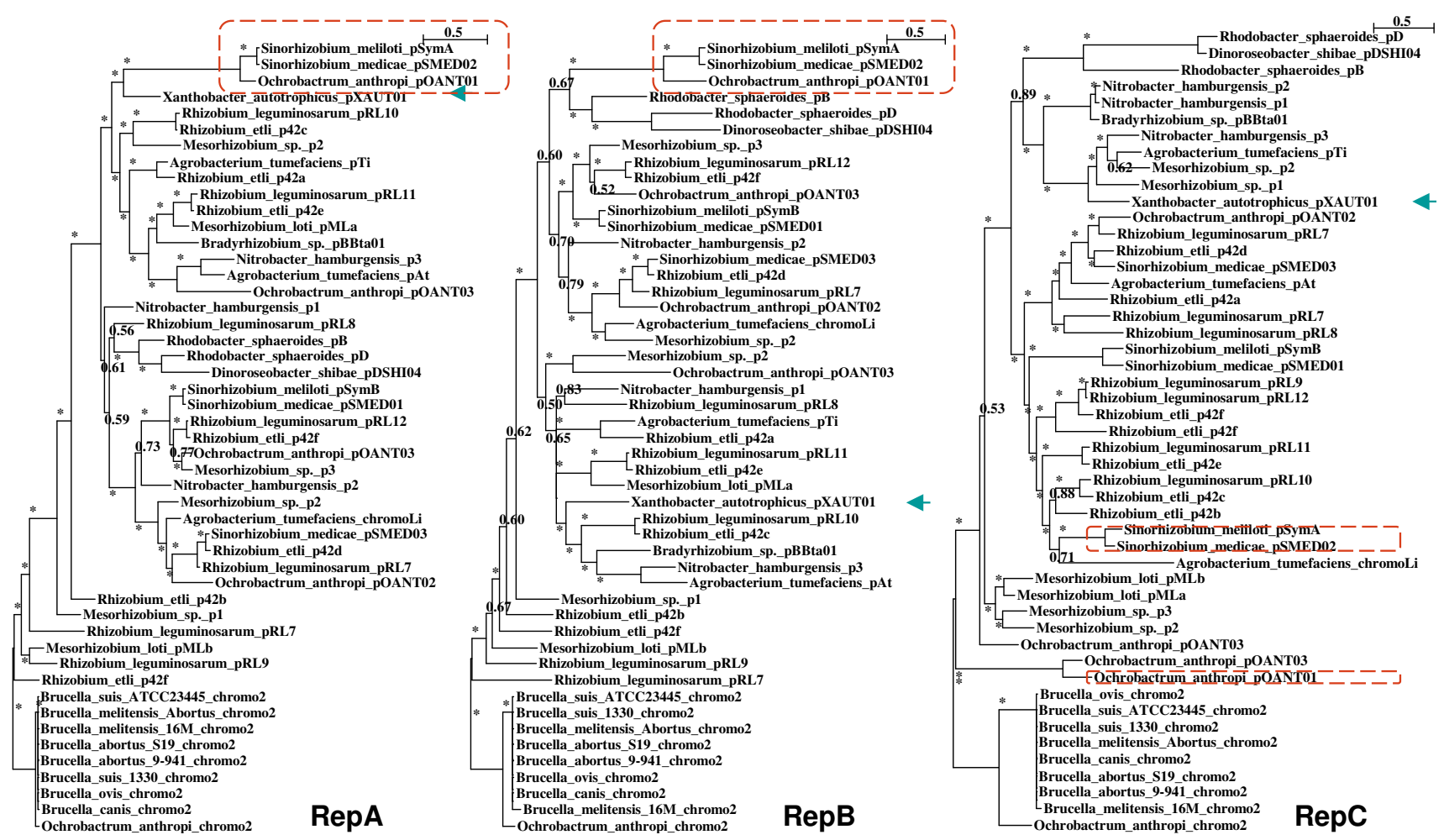

Figure I

The individual Bayesian phylogenies. The Bayesian phylogenies for RepA, RepB, and RepC. The scale bar denotes the estimated number of amino acid substitution per site. The asterisks on the branches represent posterior probability values higher than 0.95 , otherwise values are shown. All of the phylogenies were artificially rooted with the homologous gene on chromosome 2 of Ochrobactrum anthropi, to facilitate visual comparison between phylogenies. 


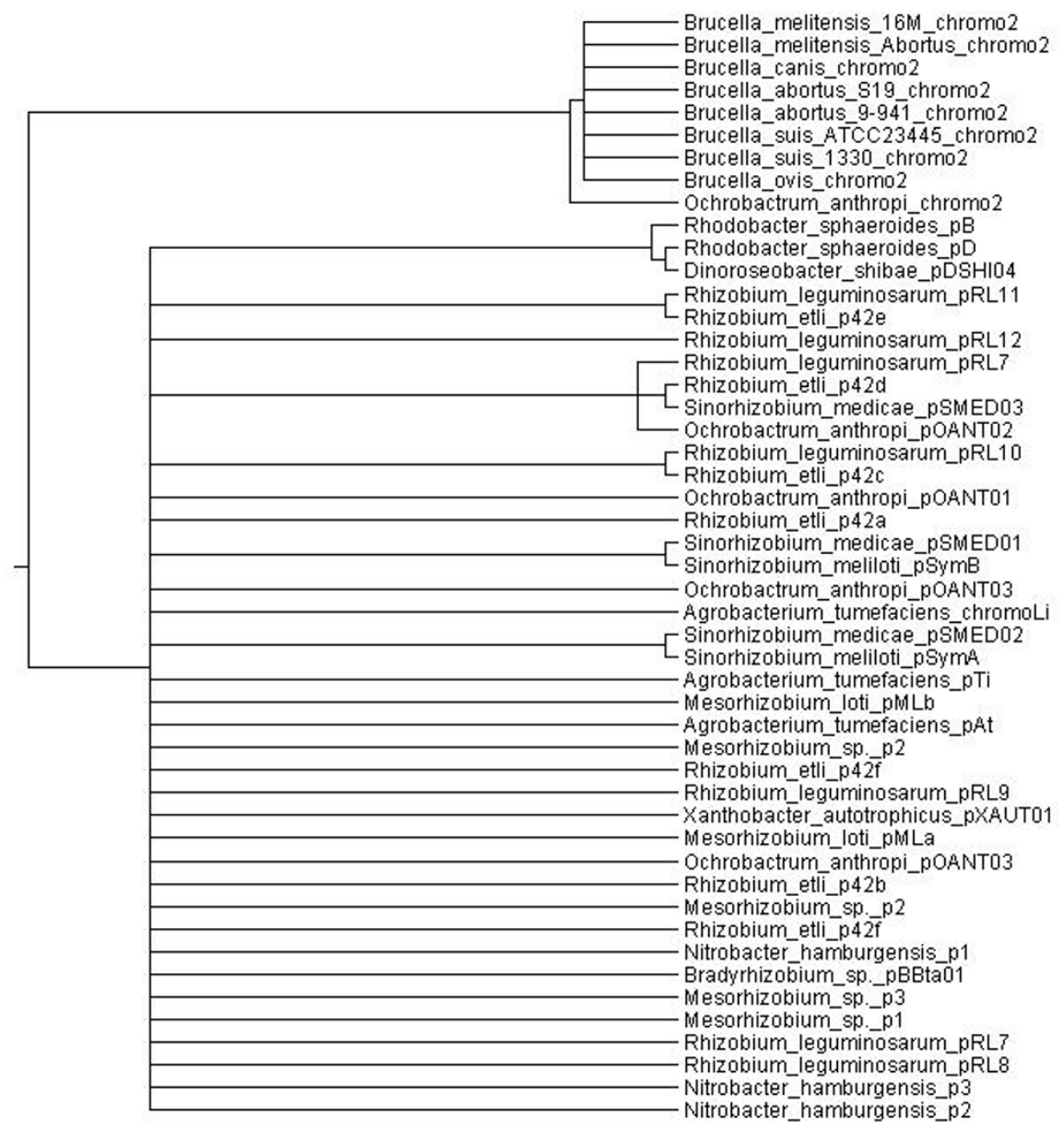

Figure 2

The strict consensus tree. A strict consensus tree was constructed using the Bayesian phylogenies of RepA, RepB, and RepC.

below 0.95 (see Figure 1). There was a large degree of conflict among the individual phylogenies, as demonstrated by the fact that the strict consensus tree had many polytomies and was poorly resolved (Figure 2). Only $25 \%$ of the nodes composing each phylogeny were shared among the three phylogenies. Since confidence sets of genes trees have been used to compare competing gene trees [13], we used this method to examine whether the differences among the phylogenies of RepA, RepB, and RepC were more than would be expected by chance (see Methods). The individual alignments of each protein rejected all but its own phylogeny, indicating that the phylogenies of the different proteins were significantly different from each other. Therefore, horizontal gene transfer has affected the individual genes within the operon. Actually each gene has had many unique horizontal gene transfer events since protein alignments rejected all but its own phylogeny. Here we will describe the positions of a couple plas- mids in the rep phylogenies to make this clear. First example, the proteins coded by genes of the repABC operon located on plasmid pXAUT01 of Xanthobacter autotrophicus occupy drastically different positions in all 3 phylogenies (see Figure 1, green arrows). Actually, in each rep phylogeny pXAUT01 clusters with distinct groups, with very good support in every case. In other example, the horizontal gene transfer has affected either 2 of the genes or one gene, as 2 phylogenies agree while the third disagrees; for example, whereas the plasmids pSymA and pSMED02 cluster with pOANT01, in RepA and RepB phylogenies, plasmid pOANT01 does not cluster with the other 2 in RepC phylogeny (see Figure 1, red squares). The horizontal gene transfer events that have affected the rep genes are very particular, as they did not disrupt the operon structure. Gene displacement in situ is the most probable process behind this observation given that the operon is conserved in all the cases. As expected, the phy- 
logenies for RepA and RepB, whose genes are next to each other, were more alike to one another than to RepC, as the Robison-Fould distance (a metric used to compare phylogenies, in which increasing distance indicates increasing disparity between phylogenies) between the phylogenies of RepA and RepB was smaller than that between RepC and either RepA or RepB (see Additional file 2). Since the evolutionary distance within the RepA, RepB, and RepC phylogenies is not that vast (see Figure 1), we checked if in situ gene displacement occurred by means of homologous recombination. To see if this might be the case here, we performed recombination analyses on the DNA alignments. In all three genes we found evidence of recombination, pairwise identity plots of the localized recombination events are presented in Additional file 3. We identified one event for repA, two for $r e p B$, and up to four for repC (see Additional files 3 and 4). The above results suggest that in situ gene displacement within the operon, through homologous recombination, has affected the repABC operon.

\section{Different levels of functional restriction within and between Rep proteins}

The most common method for modeling the variation of evolutionary rates among sites is the gamma distribution. Its shape parameter, $\alpha$, determines the extent of rate variation among sites; a small $\alpha$ represents extreme rate variation, while a large $\alpha$ value represent a minor variation in rate [14]. Given that the main reason for the heterogeneity of evolutionary rates among sites seems to be differences in their selective constraints (due to the functional and/or structural requirements of the gene), we herein used the shape parameter $\alpha$ as a proxy for the functional restriction of each studied protein. In addition, we used the total length of each phylogeny as a proxy to examine the level of protein conservation. Among the three studied proteins, RepA showed the lowest total phylogenetic length and the highest among-site rate variation (reflected through the smallest shape parameter $\alpha$ ), indicating that RepA was the most conserved protein, and that it experienced the highest level of functional restriction. The confidence intervals of the total length of the RepA phylogenies did not overlap with those of the two other phylogenies (see Table 1). Interestingly, the among-site rate variation was not significantly different between RepA and RepC, but the among-site rate variations of these two proteins were significantly different from that of RepB (see Table 1, shape parameter $\alpha$ column). Therefore, although RepA was the most conserved protein, RepA and RepC had similar levels of functional restriction.

To assess functional restriction inside the proteins, we next identified domains using Pfam [15], and assigned substitutions rates for individual sites for each protein using a discrete-gamma distribution (see Methods). We found that different domains had different substitution rates. For instance, in RepA protein, the ATPase domain almost did not have positions with highest substitution rates (see Figure 3, dotted lines, family MipZ), whereas the nucleotide-binding domain did have positions with the highest substitution rates (see Figure 3, domain CbiA). Similarly, most of the sites in the ParB-like nuclease domain of RepB (see Figure 3, dotted lines, family ParBc) had substitution rates that were smaller than those of the plasmid partition family domain (see Figure 3, family RepB). Only one domain was identified for RepC, but the substitution rates of its sites varied (Additional file 5). Notably, whereas RepA (the most conserved protein) was affected by a recombination event within its more variable domain (see Figure 3, Recombination, upper panel), RepB seems to have been affected by recombination throughout its sequence (see Figure 3, Recombination, lower panel). Thus, we detected different levels of functional restriction not only between the studied proteins, but also within them.

\section{Well differentiated incompatibility groups}

We used Rhizobium etli CFN42 and Rhizobium leguminosarum 3841 to compare and contrast incompatibility groups, because these strains each harbored six repABC compatible plasmids (i.e., six incompatibility groups). We made four DNA alignments, one for each rep gene and one for the intergenic region between the genes, which encodes a small antisense RNA gene that acts as a strong incompatibility factor. To evaluate the degree of distinction among the rep genes and intergenic region

Table I: Estimates of the best amino acid models for the individual Bayesian phylogenies

\begin{tabular}{|c|c|c|c|c|}
\hline Protein & $T L$ & Shape parameter $\alpha$ & P. Invariant sites & Model \\
\hline RepA & $11.987(11.128$ I2.906) & $0.933(0.776$ I.102) & $0.065(0.0220 .107)$ & WAG (PP I) \\
\hline RepB & $19.952(18.61721 .323)$ & I.72। ( 1.487 I.983) & $0.0698(0.04 \mid 0.103)$ & WAG (PP I) \\
\hline RepC & 17.678 (|6.49 |8.922) & I.I22 (0.993 I.265) & $0.068(0.0400 .098)$ & JTT (PP I) \\
\hline
\end{tabular}

The amino acid matrix with the highest posterior probability, the estimated proportion of invariable sites, and the estimated gamma shape parameter for each Rep protein.

Abbreviations. TL: total length of the phylogeny, PP: posterior probability. The values in parentheses is the $95 \%$ Cred. Interval. 


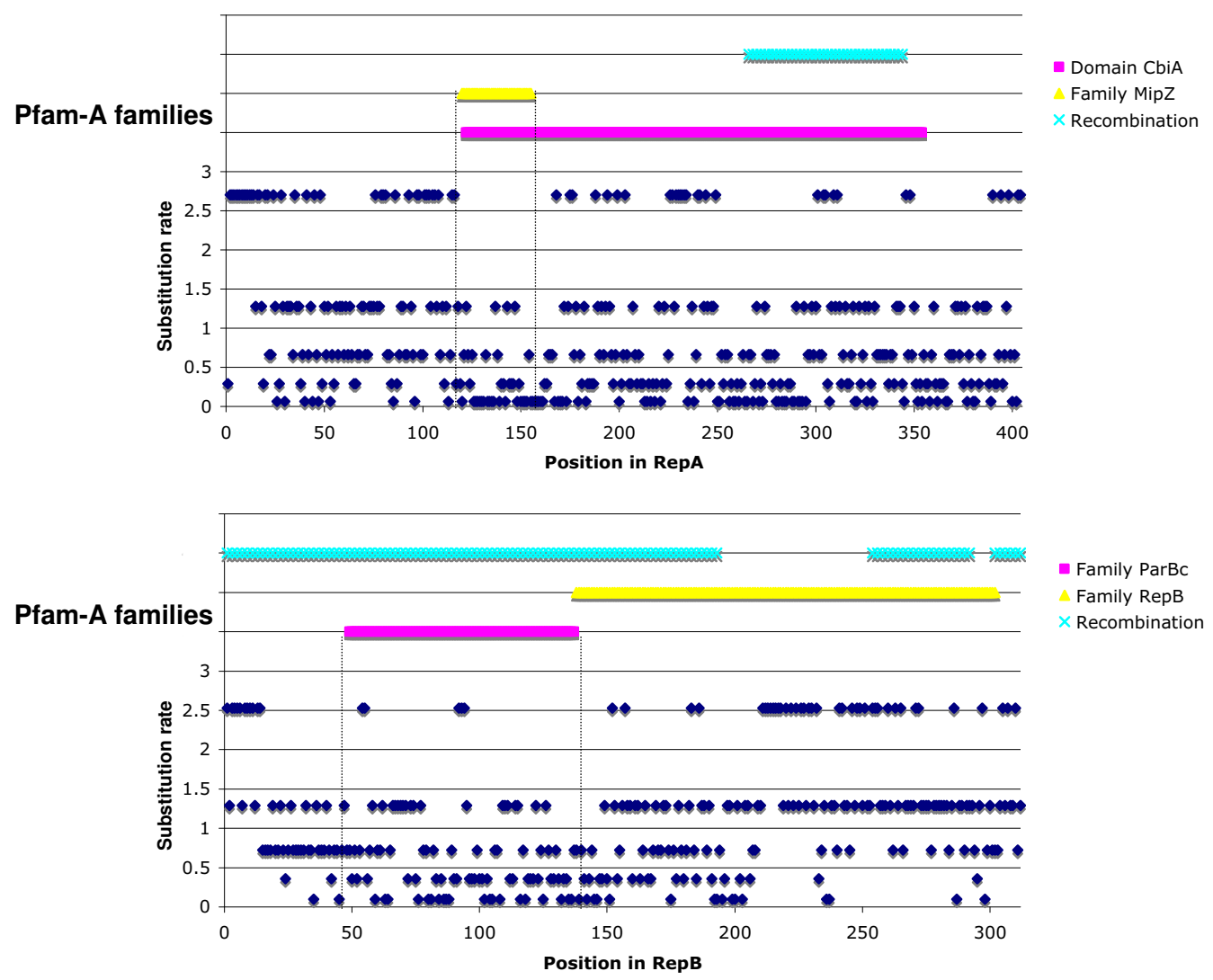

Figure 3

Functional restrictions within the RepA and RepB proteins. Substitution rate variation among sites in the RepA and RepB proteins. For each protein, all sites were assigned to one of five gamma categories. The Pfam-A domains are shown for each protein, as well as the zones affected by recombination events.

of the different incompatibility groups, we determined maximum likelihood matrices and then calculated the average distance over all possible pairs of sequences (see Methods). The genes and intergenic region could be clearly differentiated across the different plasmids (see Table 2). In agreement with our protein phylogenies, the repA and repC genes presented shorter average distances and higher proportions of invariant sites compared to

Table 2: Average between-locus distance for the different loci

\begin{tabular}{llll}
\hline Locus & Average distance+ & P. Invariant sites & Shape parameter $\alpha$ \\
\hline repA & 0.72530 & 0.194 & 1.176 \\
\hline repB & 1.13479 & 0.089 & 1.661 \\
\hline Intergenic region & 0.45827 & 0.0 & 0.47 \\
\hline repC & 0.59197 & 0.186 & 1.108 \\
\hline
\end{tabular}

The average distance over all possible sequence pairs for each locus, along with the specifications made by jModelTest regarding the substitution model.

+Average distance over all possible pairs of sequences.

All the loci but the "Intergenic region" selected the GTR model with correction for across site rate variation and invariant sites (GTR+I+G). The

"Intergenic region" selected $* T P M 2$ with correction for across site rate variation (TPM2+G).

*This model implies AC = AT; CG = GT; AG = CT; 
repB. Notably, the intergenic region comprised the shortest distance, but did not have any invariant position (see Table 2). Moreover, this locus had the highest among-site rate variation, as reflected in the smallest shape parameter $\alpha$ (see Table 2 ). This suggests that the intergenic region is under higher functional restriction compared to the rep genes; this finding is compatible with the presence of the small antisense RNA-encoding sequence in the intergenic region. Neither the intergenic region nor the rep genes showed any evidence of recombination. These results suggest that there was a high degree of differentiation among the examined incompatibility groups.

\section{Codon Adaptation Index as a measure of evolutionary adaptedness}

The Codon Adaptation Index (CAI) is a simple measure of synonymous codon usage bias. This index uses a reference set of highly expressed genes to assess the relative merits of every codon, and then determines a score for the gene or genes in question based on the use frequency of all codons in that gene [16]. The CAI can be used to evaluate the extent to which selection has been effective in molding the pattern of codon usage [16], and compare the codon usage of foreign genes versus that of highly expressed native genes. Here, we used the CAI to assess the adaptation of the repA, repB, and repC genes to their host genomes. We first calculated the relative synonymous codon usage values of highly expressed native genes (those encoding ribosomal proteins from each species), and then used CAI to compare the codon usage of the repA, repB, and repC genes to those of the reference genes (see Methods). CAI values can range from 0 (reflecting equal use of synonymous codons) to 1 (reflecting the strongest bias, codon usage is equal to that in the reference ribosomal protein-encoding genes). We found a clear trend in the CAI values within and between the studied repABC operons. In general, repA genes had the highest CAI values, followed by repB genes (see Figure 4 ). The repABC operons located on different plasmids had different CAI values, with those located on plasmids appearing to be the newest (e.g., p42a and p42d in R. etli CFN42, see Discussion) having the smallest CAI values (see Figure 4,
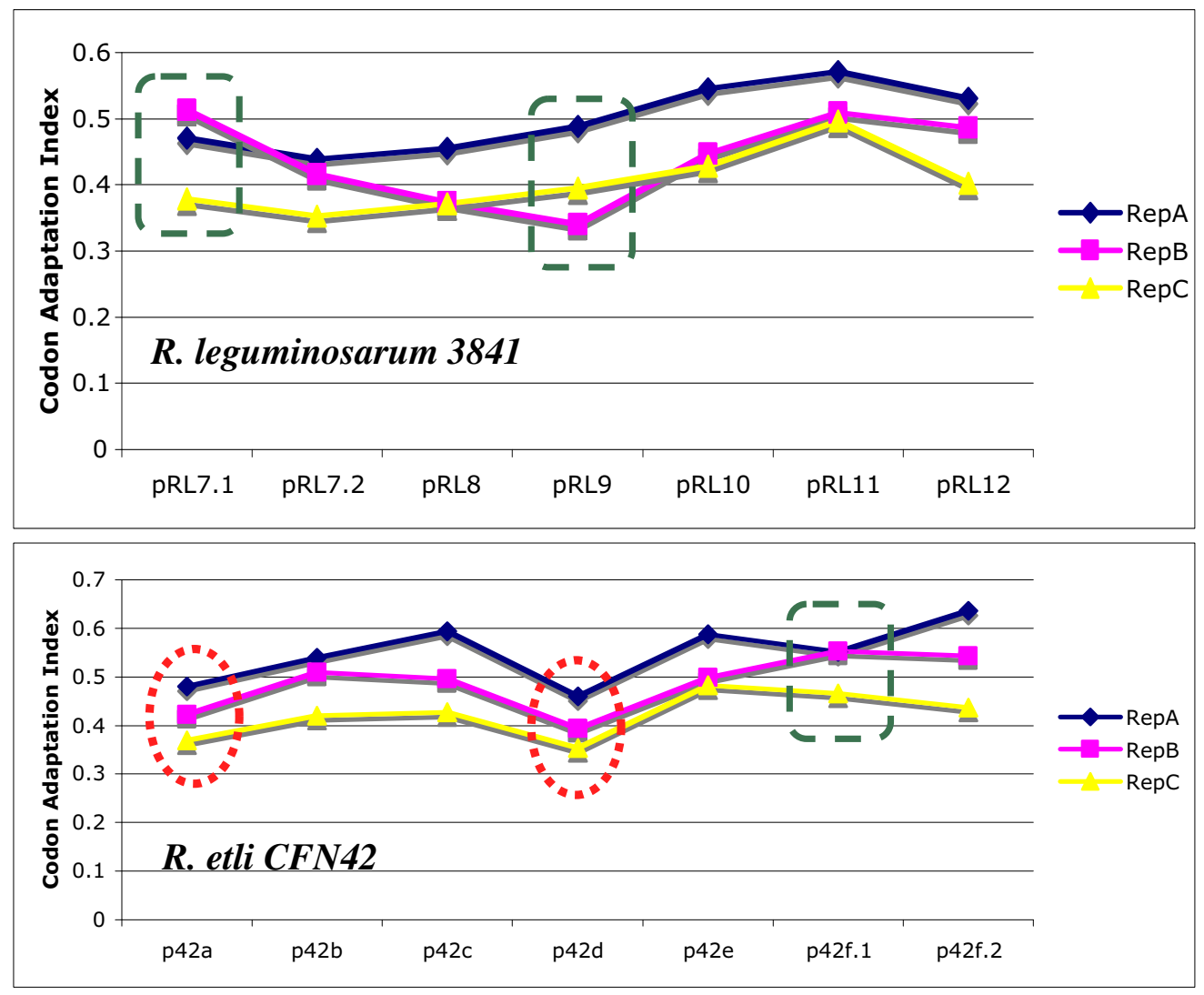

\section{Figure 4}

Codon Adaptation Index. CAI values are shown for each of the genes comprising the repABC operons found in $R$. etli CFN42 and $R$. leguminosarum 384I. Red circles indicate the putatively newest plasmids in $R$. etli CFN42. Green squares show the inconsistencies found herein. 
red circles). Notably, in plasmids harboring two repABC operons, one always failed to meet the abovementioned pattern of CAI stratification. For example, plasmid pRL7 from R. leguminosarum 3841 contained the pRL7.1 and pRL7.2 operons, and the former had a higher CAI value for repB than repA (see Figure 4, green squares). Given that the degree of codon bias in unicellular organisms correlates with the level of gene expression, our results suggest that repA is more highly expressed than the other two genes, and repB is expressed at a higher level than repC. Furthermore, it seems that the different operons have different levels of expression.

\section{Discussion}

The repABC operon is not only important because it is the replication-partition system of repABC plasmids, a common component of Alphaproteobacteria species, but because it is also the replication-partition system of some secondary chromosomes in Alphaproteobacteria species. Our present analyses functioned at two levels: within the repABC operon and between $r e p A B C$ operons in those cases where several repABC operons coexisted in the same genome. We did not find a single history within the repABC operon; clearly, each protein had its own phylogeny. This is somewhat surprising, since $\operatorname{rep} A$, repB, and repC form an operon, and it would seem that they should have similar histories if the entire operon had been horizontally transferred. Instead, even RepA and RepB, which compose the partition system and physically interact, had different phylogenies. This contrast with a recent work in which relaxase sequences were used as tools for classification of conjugative systems. In that study it was found that relaxases and the IV coupling proteins (T4CP), which map next to each other and belong to a minimal gene set that allows plasmid to be conjugally transmitted, evolve congruently for long periods of time [17]. Thus, it seems that compared with some elements of the transfer machinery the repABC replication-partition system is highly diverse.

Quite notably, every single gene of this operon presented evidence of horizontal gene transfer. In situ gene displacement is a likely process behind this, since the structure of the repABC operon is completely conserved. We think in situ gene displacement could have occurred through homologous recombination, as we found homologous recombination events across the 3 rep genes. Although in situ gene displacement appears unlikely, there is evidence that shows that this process is not that scarce. Omelchenko et al found that within the bacterial operons they had analyzed in situ gene displacement was a frequent event [12]. A striking difference between in situ gene displacement and other types of horizontal gene transfer events is that the former leaves intact the operon structure, so that, the operon is completely functional.
The proteins differed not only at the topological level, but also at the level of functional restriction. RepA and RepC, which belong to different systems, were under similar levels of functional restriction, suggesting that key elements of the partitioning and replication systems are under similar functional restrictions. In contrast, RepB had a very different level of functional restriction. We also found different levels of functional restrictions within proteins. For example, the ATPase domain of RepA (Figure 3, family MipZ), which forms a complex with the chromosome partitioning protein and is indispensable for partitioning, presented the lowest substitution rates. As well the only recombination event presented in repA did not affect the ATPase domain but a relatively unconserved part of the gene. Therefore, it seems that the different proteins, and even the different parts of the proteins themselves, are under different functional and/or structural constraints. Of the three genes studied, repA was the most conserved and might have the highest expression level. This is not unexpected, as RepA is known to have several functions, and its expression is required in both the presence and absence of partition, suggesting the need for high-level translation in order to maintain sufficient RepA levels. In contrast, repC, which is a replication initiator protein, had the lowest CAI values, perhaps due to the higher levels of homologous recombination in this gene (see below). Horizontal gene transfer could be very important in allowing the variability of this operon. Indeed, if horizontal gene transfer had not affected the genes within the operon, these genes would have to have a single evolutionary history. Instead, we found that the reverse was true. The proteins encoded in those genes not only presented different phylogenies, they also had different functional restrictions, even within the proteins themselves, and the CAI values differed among the genes. Given the presence of differences at several levels, it is very logical to think that horizontal gene transfer has unconnected the various portions of the operon, allowing each part to have a particular evolutionary history. In this way, genes with very different functional restrictions could be located next to each other, as seen for repB and repA.

The existence of multiple repABC operons located on different replicons in the same genome implies the presence of different incompatibility groups. We herein showed that when multiple repABC operons coexisted in the same genome, they were well differentiated from one another. We did not find evidence of homologous recombination in these cases; this is not unexpected, since homologous recombination would homogenize the sequences, meaning that the different groups would no longer be compatible with each other. The intergenic region, which encodes a small antisense RNA (a very important determinant for incompatibility), was highly conserved and found to be under high functional restriction, yet it did not have any 
invariant sites. Although this sequence has changed only minimally due to functional restrictions, it has still accumulated sufficient changes to allow the coexistence of the different incompatibility groups. In agreement with our within-operon analysis, repA and repC were highly conserved, with repC being the most highly conserved between operons (it had the smallest average distance). As mentioned above, repC also had the most homologous recombination events. This suggests that homologous recombination might be reducing the divergence of repC, potentially also explaining the low CAI values for this gene (homologous recombination would be erasing any improvement in the CAI values). In a report on the genome sequence of $R$. leguminosarum, Young and coworkers suggested that a recent recombination event had taken place, and divergence of RepC was not critical for plasmid compatibility [3]. Here, one of the recombination events detected in repC involved the sequence from pRL8, which is a plasmid of $R$. leguminosarum 3841.

Different $r e p A B C$ operons had distinct levels of adaptation to their host genome, with no two repABC operons presenting the same CAI values. We think that amelioration might be playing a role in the adaptation of repABC operons to their hosts. Plasmids $\mathrm{p} 42 \mathrm{a}$ and $\mathrm{p} 42 \mathrm{~d}$ were suggested to be newly acquired plasmids based on their lower GC values, poor conservation, and poor functional connectivity with the rest of the genome [4]. These two plasmids had the worst CAI values, implying that they are not well adapted to their host's genome. In contrast, the operon from $\mathrm{p} 42 \mathrm{f}$, which appeared to be the oldest plasmid harbored within R. etli CFN42, had the highest CAI values, suggesting that this operon is highly adapted. These findings indicate that the longer a repABC operon coexists with its host genome, the more adapted the operon becomes. This may result in more effective replication and partitioning processes. As well plasmids, which had the most adapted operons, presented essential genes as well; for instance plasmids pRL11, pRL12, and pRL10, which all have essential genes [3], had the operons with higher CAI values than the rest of plasmid of R. leguminosarum 3841.

\section{Conclusion}

In summary, we herein report finding different histories and functional constraints within the repABC operon. In addition, when multiple rep $A B C$ operons were present in the same genome, they had different levels of adaptedness to the host genome, and this seems to be related to the length of time each operon had been associated with the host genome. Finally, horizontal gene transfer with conservation of the operon structure provides a highly dynamic operon in which each member could have its own evolutionary dynamics.

\section{Methods}

\section{Detection of homologous genes and operons}

We first identified the homologous of the RepA, RepB, and RepC proteins across the known Alphaproteobacteria genomes (see Additional file 6). The RepA, RepB, and RepC proteins from symbiotic plasmids of $R$. etli CFN42 and $S$. meliloti 1021 were used as seeds, and were queried against the proteomes encoded by the other genomes (Additional file 6), using BLAST [18] with an E-value cutoff of 1.0e-12. We retained all cases where a seed protein had a hit in any other proteome and the proteins aligned along at least $70 \%$ of their lengths. We then selected for DNA sequences wherein repA was next to $r e p B$, and $r e p B$ was next to repC (by definition, the only gene between repA and repC was repB), this was taken as a complete operon. The homologous protein groups contained only proteins whose genes formed complete operons. For each homologous protein group, we constructed an alignment with MUSCLE [19], and used this alignment to infer a phylogeny (see below). To generate the DNA alignments of $r e p A, r e p B$, and $r e p C$, we used their protein alignments as references, and performed nucleotide alignment using the "tranalign" program from The European Molecular Biology Open Software Suite (EMBOSS) [20]. The recombination analysis was carried out on these DNA alignments.

Other sets of DNA alignments were created for each of the operons contained in R. etli CFN42 and R. leguminosarum 3841. The intergenic region between $r e p B$ and $r e p C$ was also considered. We then used jModelTest [21] to carry out statistical selection of the best-fit models of nucleotide substitution for every DNA alignment. Finally, maximum likelihood distance matrices were inferred using the model specifications from jModelTest; this was done with PUZZLE [22].

\section{Phylogenetic Analysis}

Phylogenies were created using MrBayes v3.1.2 [23], allowing the MCMC sampler to explore all of the fixedrated amino acid models included in MrBayes. The number of rate categories for gamma distributions was set to four, with a proportion of sites allowed to be invariable. We performed two runs with four chains each, for $5,000,000$ generations. Trees were sampled every 1000 generations, $20 \%$ of all generations were removed as burn-in, and a consensus tree was taken. We also estimated the best amino acid models, including the amino acid matrices with the highest posterior probability, estimates of the proportion of invariable sites, and estimates of the gamma shape parameter.

A strict consensus tree was created from all three Bayesian phylogenies, using CONSENSE [24]. 
We established the similarities of the phylogenies using the Robinson and Fould distance (RFd), as calculated with TREEDIST [24].

We used confidence sets to assess whether the differences in topology between the individual Bayesian phylogenies exceeded those expected to occur by chance. We used expected likelihood weighting [13], which provides a simple and intuitive method for making multiple comparisons of models and constructing the corresponding confidence sets. This test has the benefit of being less conservative than the Shimodaira-Hasegawa test [13]. The topologies tested included those from the RepA, RepB, and RepC phylogenies. PUZZLE [22] was used to carry out this test for each protein alignment.

\section{Recombination analysis}

Although methods that use the substitution patterns or incompatibilities among sites seem be the most powerful strategy for identifying the presence of recombination events, no single method seems to perform optimally under all different scenarios [25]. Thus, the best strategy is often to use a combination of methods. Here, we used the RDP3 program [26], which implements a number of methods for identifying recombination events, including GENECONV [27], RDP [26], MaxChi [28], Chimera [28], SisCan [29], and Bootscanning [30]. We identified a recombination event as valid when at least three of the six methods indicated positive findings.

\section{Functional regions and among-site rate variation in $\operatorname{Rep}$ proteins}

We identified the various protein domains by applying the Pfam-A component of Pfam [15]. For this analysis, the RepA, RepB, and RepC proteins of symbiotic plasmid p42d from R. etli CFN42 were queried against Pfam-A. For every position of each protein alignment, a substitution rate was assigned using a discrete-gamma distribution. The discrete-gamma distribution used five rate classes and was implemented through PUZZLE.

\section{Codon Adaptation Index as measure of evolutionary adaptedness}

This analysis was done only for the repA, repB, and repC genes located on operons found within species $R$. etli CFN42 and R. leguminosarum 3841. We used the utility "cusp" from EMBOSS to calculate a codon usage table for the genes encoding the ribosomal proteins in each species. Using these tables as a reference, we applied the "cai" program of the EMBOSS suite to calculate Codon Adaptation Indices for the rep $A$, repB, and rep $C$ genes.

\section{Authors' contributions}

SC-R conceived and designed the experiments. SC-R and JFV-C performed the experiments. SC-R analyzed the data.
SC-R and MAC discussed the results. MAC and SC-R wrote the manuscript. JFV-C and VG checked the manuscript. VG contributed materials. All authors read and approved the final manuscript.

\section{Additional material}

\section{Additional file 1}

Homologous genes and repABC operons. Homologous genes of repA, repB, and repC, as well as complete and faulty repABC operons found across the studied Alphaproteobacteria genomes. For each gene it was registered whether it was located on a chromosome (C) or a plasmid (P). Click here for file

[http://www.biomedcentral.com/content/supplementary/14712164-10-536-S1.DOC]

\section{Additional file 2}

Robison-Fould distances between Rep phylogenies. In order to determine the similarity among the Rep phylogenies, Robison-Fould distances between Rep phylogenies were established.

Click here for file

[http://www.biomedcentral.com/content/supplementary/14712164-10-536-S2.DOC]

\section{Additional file 3}

Recombination events identified for repA, repB, and repC. Pairwise identity plots of the localized recombination events, showing major and minor parent sequences as well as the daughter sequence. Abbreviations are given in Additional file 4.

Click here for file

[http://www.biomedcentral.com/content/supplementary/14712164-10-536-S3.PPT]

\section{Additional file 4}

Abbreviations of additional file 3. Abbreviations used in the pairwise identity plots in additional file 3 .

Click here for file

[http://www.biomedcentral.com/content/supplementary/1471-

2164-10-536-S4.DOC]

\section{Additional file 5}

Functional restrictions within RepC. Substitution rate variation among sites in RepC. All sites were assigned to one of five gamma categories. Pfam-A domains are shown, as well as the zone affected by recombination events.

Click here for file

[http://www.biomedcentral.com/content/supplementary/1471-

2164-10-536-S5.PPT]

\section{Additional file 6}

Genomes used. Alphaproteobacteria genomes used to search for repABC operons.

Click here for file

[http://www.biomedcentral.com/content/supplementary/14712164-10-536-S6.DOC] 


\section{Acknowledgements}

We thank Luis Lozano for his comments on the manuscript. SC-R acknowledges a PhD fellowship from CONACyT, and thanks the Computational Unit of the Instituto de Biotecnología, particularly Jerome Verleyen, and the "Macroproyecto de Tecnologías de la Información y la Computación de la UNAM," for the use of their computer facilities. This work was partially supported by PAPIIT Grant IN205808. SC-R would like to extend warm thanks to Valeria Lobos for all the non-academic support, which is the one that most counts.

\section{References}

I. Cevallos MA, Cervantes-Rivera R, Gutierrez-Rios RM: The repABC plasmid family. Plasmid 2008, 60(I): 19-37.

2. Pappas KM: Cell-cell signaling and the Agrobacterium tumefaciens Ti plasmid copy number fluctuations. Plasmid 2008, 60(2):89-107.

3. Young JP, et al:: The genome of Rhizobium leguminosarum has recognizable core and accessory components. Genome Biol 2006, 7(4):R34.

4. Gonzalez $V$, et al: The partitioned Rhizobium etli genome: genetic and metabolic redundancy in seven interacting replicons. Proc Natl Acad Sci USA 2006, I03( I 0):3834-9.

5. Kumar CC, Novick RP: Plasmid pTI8I replication is regulated by two countertranscripts. Proc Natl Acad Sci USA 1985, 82(3):638-42.

6. MacLellan SR, et al.: The expression of a novel antisense gene mediates incompatibility within the large repABC family of alpha-proteobacterial plasmids. Mol Microbiol 2005, 55(2):6II-23.

7. Slater SC, et al:: Genome sequences of three agrobacterium biovars help elucidate the evolution of multichromosome genomes in bacteria. J Bacteriol 2009, I 9 I(8):250 I-I I.

8. Chai Y, Winans SC: RepB protein of an Agrobacterium tumefaciens Ti plasmid binds to two adjacent sites between repA and repB for plasmid partitioning and autorepression. Mol Microbiol 2005, 58(4): I I 14-29.

9. Ramirez-Romero MA, et al.: Structural elements required for replication and incompatibility of the Rhizobium etli symbiotic plasmid. J Bacteriol 2000, I82(I I):3 I I7-24.

10. Venkova-Canova T, et al:: Two discrete elements are required for the replication of a repABC plasmid: an antisense RNA and a stem-loop structure. Mol Microbiol 2004, 54(5): I43I-44.

II. Cevallos MA, et al: Rhizobium etli CFN42 contains at least three plasmids of the repABC family: a structural and evolutionary analysis. Plasmid 2002, 48(2): 104-16.

12. Omelchenko MV, et al.: Evolution of mosaic operons by horizontal gene transfer and gene displacement in situ. Genome Biol 2003, 4(9):R55.

13. Strimmer K, Rambaut A: Inferring confidence sets of possibly misspecified gene trees. Proc Biol Sci 2002, 269(1 487): 137-42.

14. Yang Z: Among-site rate variation and its impact on phylogenetic analyses. Trends Ecol Evol 1996, I I (9):6.

15. Finn RD, et al.: The Pfam protein families database. Nucleic Acids Res 2008:D28I-8.

16. Sharp PM, Li WH: The codon Adaptation Index--a measure of directional synonymous codon usage bias, and its potential applications. Nucleic Acids Res 1987, I 5(3): | 28 I-95.

17. Garcillan-Barcia MP, Francia MV, de la Cruz F: The diversity of conjugative relaxases and its application in plasmid classification. FEMS Microbiol Rev 2009, 33(3):657-87.

18. Altschul SF, et al.: Gapped BLAST and PSI-BLAST: a new generation of protein database search programs. Nucleic Acids Res 1997, 25(17):3389-402.

19. Edgar RC: MUSCLE: a multiple sequence alignment method with reduced time and space complexity. BMC Bioinformatics 2004, 5: II3.

20. Rice P, Longden I, Bleasby A: EMBOSS: the European Molecular Biology Open Software Suite. Trends Genet 2000, 16(6):276-7.

21. Posada D: jModelTest: phylogenetic model averaging. Mol Biol Evol 2008, 25(7): $1253-6$.
22. Schmidt HA, et al.: TREE-PUZZLE: maximum likelihood phylogenetic analysis using quartets and parallel computing. Bioinformatics 2002, 18(3):502-4.

23. Ronquist F, Huelsenbeck JP: MrBayes 3: Bayesian phylogenetic inference under mixed models. Bioinformatics 2003, 19(12): I572-4.

24. Felsenstein J: PHYLIP (Phylogeny Inference Package) version 3.6. Department of Genome Sciences, University of Washington, Seattle; 2005.

25. Posada D, Crandall KA, Holmes EC: Recombination in evolutionary genomics. Annu Rev Genet 2002, 36:75-97.

26. Martin DP, Williamson C, Posada D: RDP2: recombination detection and analysis from sequence alignments. Bioinformatics 2005, 2 I (2):260-2.

27. Padidam M, Sawyer S, Fauquet CM: Possible emergence of new geminiviruses by frequent recombination. Virology 1999, 265(2):2। 8-25.

28. Smith JM: Analyzing the mosaic structure of genes. J Mol Evol 1992, 34(2): 126-9.

29. Gibbs MJ, Armstrong JS, Gibbs AJ: Sister-scanning: a Monte Carlo procedure for assessing signals in recombinant sequences. Bioinformatics 2000, 16(7):573-82.

30. Salminen $\mathrm{MO}$, et al.: Identification of breakpoints in intergenotypic recombinants of HIV type I by bootscanning. AIDS Res Hum Retroviruses 1995, I I(I I): I423-5.
Publish with Bio Med Central and every scientist can read your work free of charge

"BioMed Central will be the most significant development for disseminating the results of biomedical research in our lifetime. "

Sir Paul Nurse, Cancer Research UK

Your research papers will be:

- available free of charge to the entire biomedical community

- peer reviewed and published immediately upon acceptance

- cited in PubMed and archived on PubMed Central

- yours - you keep the copyright

Submit your manuscript here:

http://www.biomedcentral.com/info/publishing_adv.asp
BioMedcentral 